\title{
How to improve low lymph node recovery rates from axillary clearance specimens of breast cancer. A short term audit
}

\author{
Gábor Cserni
}

\begin{abstract}
Aim-To implement an audit scheme to increase the lymph node yield from axillary clearance specimens.

Methods-Two pathologists cut up each specimen after weighing it. The number of nodes and the dimensions of the largest and smallest nodes were recorded, together with the number of non-lymph node structures recovered. Fifty consecutive audited cases were compared with $\mathbf{5 0}$ consecutive cases assessed before the audit process.

Results-It proved possible to increase the median number of lymph nodes from 10 to 22. There was an obvious learning period, during which the number of nodes recovered during the second pathologist's cut-up gradually decreased, while the total number remained relatively constant. The increase in lymph node yield resulted from the recovery of smaller nodes. The identification of lymph nodes also improved, and fewer non-lymph node structures were recovered by the end of the study.

Conclusions-Such an audit scheme can be recommended for all institutions where the lymph node yield of axillary clearance specimens seems suboptimal. The relevance of recovering more nodes remains to be determined; from this small series, it seems to have no clinical impact.

(F Clin Pathol 1998;51:846-849)
\end{abstract}

Keywords: audit; axillary clearance; histopathology; lymph node recovery

The use of axillary surgery in the treatment of breast cancer remains controversial. Axillary clearance (total or level I-II dissection), axillary sampling, sentinel node biopsy, or omission of axillary surgery with or without irradiation to the axilla are practised in different settings, but no consensus exists. There is no doubt that all removed lymph nodes must be examined histologically, since the lymph node status is the most powerful single prognostic factor and has a significant impact on the further treatment of the patient.

It is widely accepted that a minimum of 10 lymph nodes per axillary clearance specimen must be examined for an adequate assessment of the axillary lymph node status. ${ }^{1-5}$ The number of lymph nodes examined by the histopathologist depends on (1) anatomical differences, (2) the diligence of the surgeon in removing the nodes from the axilla, and (3) the diligence of the pathologist in recovering lymph nodes from the dissection specimen. ${ }^{6}$ The first of these factors is obvious. The second has been audited and found to make a contribution to a lower recovery rate in up to $38 \%$ of the clearance procedures, but to only a minor degree. ${ }^{7}$ Our necropsy experience supports this.

The number of pathologically assessed lymph nodes varies greatly from institution to institution, with the median number of lymph nodes per axilla ranging from eight to $28 .^{6} \mathrm{I}$ made a retrospective study of the numbers of lymph nodes histopathologically assessed between 1980 and 1996. ${ }^{5}$ Despite a gradual increase in the number of nodes recovered, I found that approximately three quarters of all the specimens contained fewer than 10 lymph nodes, the median number of nodes recovered in recent years being nine per axilla. ${ }^{5}$ This is comparable with the experience in some other institutions, ${ }^{36}$ but seems suboptimal in view of the recommended minimum of 10 nodes.

Table 1 Comparison of audited and control groups

\begin{tabular}{|c|c|c|c|}
\hline Variable & Control & Audited & $p$ Value \\
\hline \multicolumn{4}{|c|}{ UICC $p T$ category of primary tumour } \\
\hline pTis & 0 & 1 & \multirow[t]{6}{*}{$\mathrm{p}>0.6, \mathrm{NS}$} \\
\hline $\mathrm{pTx}$ & 0 & 1 & \\
\hline pT1 & 13 & 17 & \\
\hline pT2 & 27 & 24 & \\
\hline pT3 & 3 & 2 & \\
\hline pT4 & 7 & 5 & \\
\hline \multicolumn{4}{|c|}{ Axillary lymph node status } \\
\hline Negative & 23 & 31 & \multirow[t]{4}{*}{$\mathrm{p}>0.1, \mathrm{NS}$} \\
\hline Positive & 27 & 19 & \\
\hline 1-3 positive & 13 & 6 & \\
\hline$>3$ positive & 14 & 13 & \\
\hline \multicolumn{4}{|c|}{ Number of recovered lymph nodes } \\
\hline Mean & 10.6 & 23.56 & \multirow[t]{3}{*}{$\mathrm{p}<0.001$} \\
\hline Median & 10 & & \\
\hline Range & $4-39$ & $11-42$ & \\
\hline \multicolumn{4}{|c|}{ Smallest lymph node dimension (mm) I: 1st cut-up only } \\
\hline Mean & 3.09 & 1.4 & \multirow[t]{3}{*}{$\mathrm{p}<0.001$} \\
\hline Median & 2.8 & 1.2 & \\
\hline Range & $0.5-8.4$ & $0.5-3.8$ & \\
\hline \multicolumn{4}{|c|}{ Smallest lymph node dimension (mm) II: 2 nd cut-up included } \\
\hline Mean & & 1.15 & \multirow[t]{3}{*}{$\mathrm{p}<0.001$} \\
\hline Median & & 1.0 & \\
\hline Range & & $0.5-3.8$ & \\
\hline \multicolumn{4}{|c|}{ Largest lymph node dimension ( $\mathrm{mm}$ ) } \\
\hline Mean & 14.78 & 16.9 & \multirow[t]{3}{*}{$\mathrm{p}>0.05, \mathrm{NS}$} \\
\hline Median & 14.85 & 16 & \\
\hline Range & $8.0-23.2$ & $7-45$ & \\
\hline \multicolumn{4}{|c|}{ Number of "non-lymph-nodes" } \\
\hline Mean & 1.5 & 9.18 & \multirow[t]{3}{*}{$\mathrm{p}<0.001$} \\
\hline Median & 1 & & \\
\hline Range & $0-6$ & $0-30$ & \\
\hline
\end{tabular}

"Smallest lymph node dimension" refers to the largest dimension of the smallest node. 


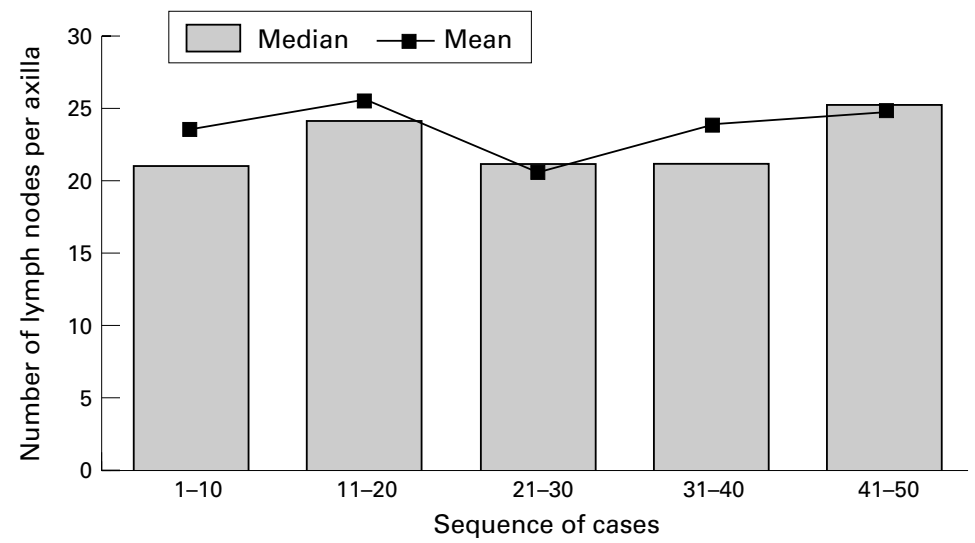

Figure 1 Lymph node yield through the audit.

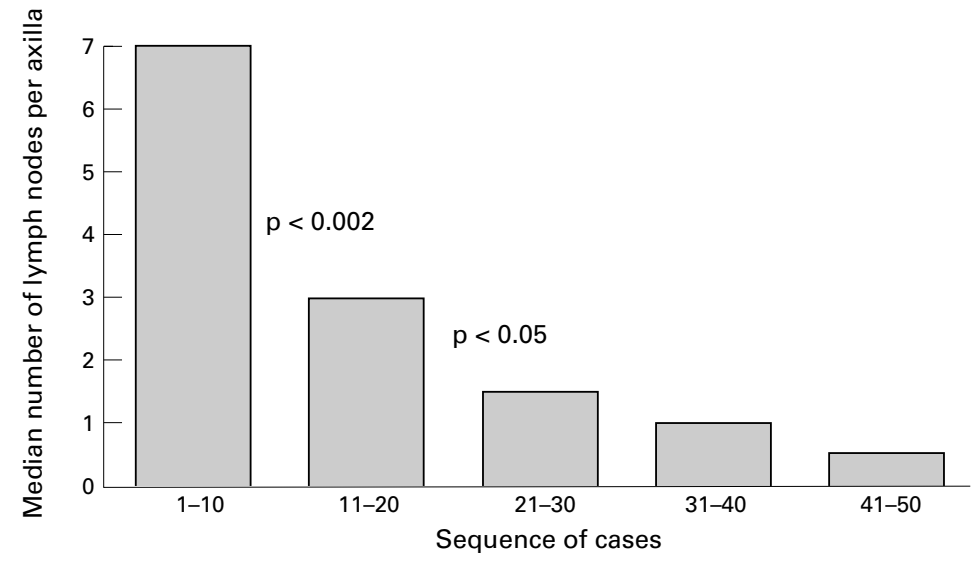

Figure 2 Median number of lymph nodes recovered during the second cut-up.

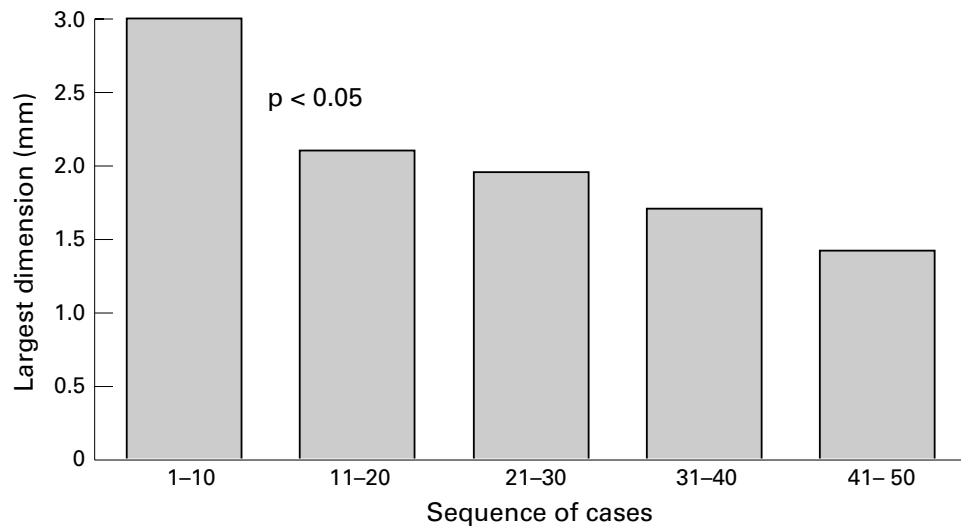

Figure 3 Median of largest dimension of lymph nodes recovered during the second cut-up.

Accordingly, I decided to monitor our lymph node recovery rate and set up audit conditions in 1997.

\section{Methods}

I audited the recovery rate of 50 consecutive axillary clearance specimens between 23 July and 12 December 1997 and compared the results with those of the 50 specimens immediately preceding those audited, also all processed in 1997; there was thus no overlap with my previous study. ${ }^{5}$ The surgical procedure used was identical in the two groups compared: axillary dissection after $\mathrm{Cady},{ }^{8}$ combined with level III dissection in the event of clinical involvement. ${ }^{9}$
The formalin fixed or fresh dissection specimens were weighed (a procedure introduced into our cut-up protocol from the beginning of the audit) and cut up, with the aid of simultaneous palpation, into thin parallel sections. Care was taken not to cut through palpable lymph nodes. All nodes were processed separately, but only a part of larger nodes was taken for histology. After this initial cut-up, the remains of these larger nodes were isolated from the axillary fat residues considered to be free of lymph nodes. A second pathologist then assessed the specimen and tried to remove as many further lymph nodes as possible. For the purpose of the audit, only two pathologists shared these duties in rotation, one or other of them always auditing the performance of the other. All lymph nodes were cut in such a manner as to obtain their largest dimension on the histological slides.

The lymph nodes were counted, and the largest dimensions of the largest and smallest nodes were measured with Vernier scales. ${ }^{10}$ The number of structures originally thought to represent lymph nodes but microscopically proving to be something else was also recorded.

Statistical comparison between groups was made with the Student $t$ test for parametric data and with the $\chi^{2}$ test for categoric data. In comparisons involving the quantitative axillary nodal status of the patients, a prognostic approach was used, the patients being divided into node negative and node positive groups, the latter category being further divided into patients with one to three involved nodes or more than three involved nodes.

\section{Results}

Most of the clearance specimens weighed between 51 and $100 \mathrm{~g}$. The median number of lymph nodes showed a tendency to increase with increasing axillary fat weight $(18,22$, and 30 for the ranges $0-50 \mathrm{~g}, 51-100 \mathrm{~g}$, and $>100$ $\mathrm{g}$, respectively; $\mathrm{n}=11,30$, and 9 patients, respectively), but no consistent relation was observed between the two variables.

The study group and the control group did not differ significantly in terms of the tumour pT category, ${ }^{11}$ the qualitative or quantitative axillary nodal status, or the largest lymph node dimension, but there was a significant difference between the two groups with regard to the number of lymph nodes, the size of the smallest lymph nodes, and the number of non-lymph-node structures removed as lymph nodes (table 1).

The total number of lymph nodes recovered did not change significantly during the audit process (fig 1), but the number removed by the auditor during the second cut-up gradually decreased (fig 2). The size of the lymph nodes removed by the auditor also showed a gradual decrease (fig 3).

The number of non-lymph-node structures reached a maximum in the second group of 10 cases, while the number of non-lymph-node structures recovered during the second cut-up showed a gradual decrease (fig 4). The ratio of the number of non-lymph-node structures to the number of lymph nodes recovered was used 


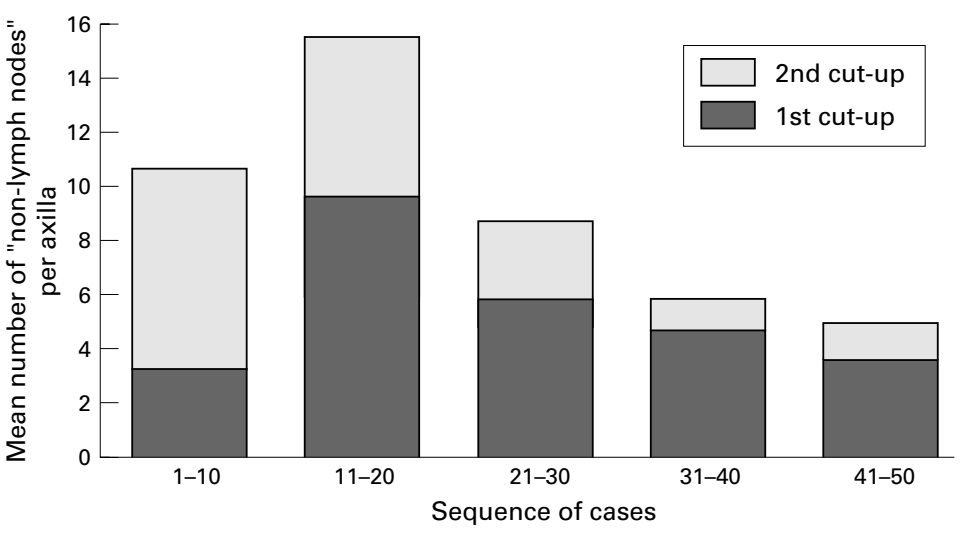

Figure 4 Non-lymph-node structures recovered through the audit.

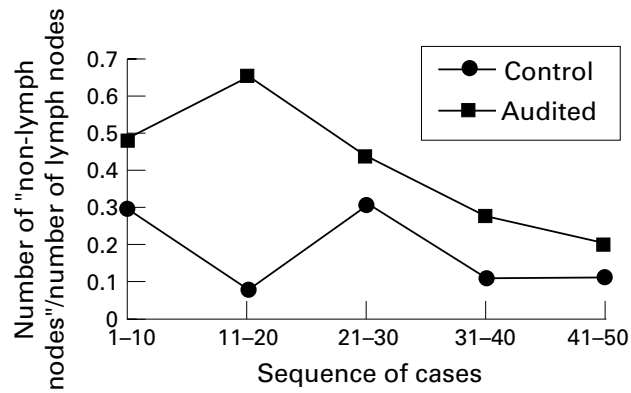

Figure 5 Ratio of number of non-lymph-node structures to number of lymph nodes through the audit.

to monitor the specificity of lymph node identification. This also showed a peak in the second group of 10 cases, but it then gradually decreased and attained the range for the control group (fig 5). Non-lymph-node structures included mainly fibro-fatty tissue (often cauterised) and vessels, but breast tissue (probably axillary tail), nerves, clots, and muscle fibres were also seen in this category.

The total number of lymph nodes removed during the second cut-up was 146 (12.4\% of all lymph nodes recovered). Only five metastatic nodes were found among these $(3.2 \%$ of all metastatic nodes). In no instance did the second cut-up alter the qualitative or quantitative axillary lymph node status; the ratio of positive nodes to all nodes changed from $10 / 19,18 / 25,27 / 40$, and $18 / 28$ to $11 / 42,20 / 33$, $28 / 42$, and $19 / 30$ in cases $4,5,35$, and 50 , respectively. All the metastases identified from the second cut-up were micrometastases, ranging in size from 0.7 to $2.0 \mathrm{~mm}$.

\section{Discussion}

We initiated an audit process to maximise the lymph node yield from axillary clearance specimens without substantially changing our method of cutting up specimens. It is generally accepted that the lymph node yield can be increased through the use of clearing substances, ${ }^{6}{ }^{12}$ but this is not considered essential or worth the time and money. Furthermore, without clearing axillary fat, some institutions can reach recovery rates as high as those attained when clearing techniques are employed. ${ }^{6}$

We have introduced weighing of the axillary fat as an objective method of describing the specimen. Although the median number of lymph nodes showed a tendency to increase with greater weight, no consistent linear relation was found between the sample weight and the number of nodes within this small sample, perhaps because of anatomical differences in the number of nodes and constitutional differences.

We achieved our target-increasing the median lymph node recovery rate per axilla from 10 to 22 . (It should be noted that 20 of our patients underwent sentinel lymph node biopsy with removal of one or two lymph nodes before axillary dissection, and these extra nodes were not included in the study.) When compared with the increase in yield resulting from axillary re-excision, ${ }^{7}$ this result clearly shows the important role of the pathologist in obtaining a larger number of lymph nodes for histological assessment. Although the total number of nodes removed from a specimen during the study period was reasonably constant, figs $2-5$ reflect a learning process. In the first 10 cases, many lymph nodes (range 4-23) were removed during the second cut-up. The number of lymph nodes recovered by the auditor showed a continuous decrease, reflecting an increase in the yield produced by the first assessor of the specimen. The size of the nodes recovered also decreased, and the difference in number between the control and study groups resulted from an increase in the number of smaller nodes, since the largest lymph node dimension was similar in the two groups. The study process therefore resulted in the identification of a larger number of small nodes. It also helped reduce the number of "false" lymph nodes.

With regard to clinical relevance, there was no significant difference between the control group and the study group in the proportion of node positive tumours. This suggests that the increase in lymph node recovery rate was not paralleled by a rise in the detection of metastatic disease. Although the two-assessor system was ideal for audit purposes, following the completion of the study period there was no further need for such a cut-up protocol, since fewer and fewer nodes were found during the second cut-up, and the nodes recovered by the auditor merely increased the total number of lymph nodes but had no clinical impact.

We share the opinion that all submitted lymph nodes should be assessed histologically and that the largest possible lymph node yield should be aimed at in axillary dissection specimens. ${ }^{12}$ Our audit proved effective in this respect and I can recommend it for those institutions where lymph node yields are low. However, the clinical impact of assessing an increased number of nodes seems minimal and, if a larger number of cases gives similar results (our ongoing retrospective data collection seems to suggest this), the cost and benefit features accompanying the recovery of more nodes must also be considered.

I thank Dr M Tarján for his contribution to the work. The study was supported by the János Bolyai fellowship of the Hungarian Academy of Sciences. 
1 Fisher B, Wolmark N, Bauer M, et al. The accuracy of clinical nodal staging and of limited axillary dissection as a determinant of histologic nodal status in carcinoma of the

2 Kjaergaard J, Blichert-Toft $M$, Andersen JA, et al, and the Danish Breast Cancer Cooperative Group. Probability of false negative nodal staging in conjunction with partial axillary dissection in breast cancer. Br F Surg 1985;72:3657.

3 Mathiesen O, Carl J, Bonderup O, et al. Axillary sampling and the risk of erroneous staging of breast cancer. Acto Oncol 1990;29:721-5.

4 Kiricuta CI, Tausch J. A mathematical model of axillary lymph node involvement based on 1446 complete axillary dissections in patients with breast carcinoma. Cancer 1992; 69:2496-501.

5 Cserni G. Changing trends in lymph node recovery from axillary clearance specimens in breast cancer. Possible implications for the quantitative axillary status from a 17 year retrospective study. Eur f Oncol 1997;2:403-8.
6 Fisher B, Slack NH. Number of lymph nodes examined and the prognosis of breast carcinoma. Surg Gynecol Obstet 1970;131:79-88.

7 Reynolds JV, Mercer P, McDermott EWM, et al. Audit of complete axillary dissection in early breast cancer. Eur $\mathcal{F}$ Cancer 1994;30A:148-9.

8 Cady B. Total mastectomy and partial axillary dissection. Surg Clin North Am 1973;53:313-18.

9 Blichert-Toft M, Smola MG, Cataliotti L, et al. Principles and guidelines for surgeons-management of symptomatic breast cancer. On behalf of the European Society of Surgical Oncology. Eur f Surg Oncol 1997;23:101-9.

10 Kirkham N, Cotton DWK. Measuring melanomas: the Vernier method. F Clin Pathol 1984;37:229-30.

11 Union Internationale Contre le Cancer. Breast tumours. In: Spiessl B, Beahrs OH, Hermanek P, et al. eds. TNM atlas, 3rd ed. Berlin: Springer-Verlag, 1990:173-83.

12 Royal College of Pathologists Working Group. Pathology reporting in breast cancer screening. $\mathcal{F}$ Clin Pathol 1991;44: reporting

\section{Royal College of Pathologists Forthcoming Symposia}

Transfusion Medicine Update

17-18 November 1998

Cytopathology Update

17 December 1998

What to do When the Cause of Death is in the Brain

27 January 1999

Antimicrobial Resistance: In Humans and Other Animals

17 February 1999

Cytokines and Chemokines in Disease

18 March 1999

Can't Give a Cause of Death-Won't Give a Cause of Death 22 April 1999

These meetings will be held at the Royal College of Pathologists, 2 Carlton House Terrace,

London SW1Y 5AF and are open to members and non-members of the College.

Further details and application forms from the Scientific Meetings Officer, tel +44 (0)171930 5862, ext 24/25; fax +44 (0)1713210523. 